\title{
Newer optics efficiently mix, dim, and color-tune LED light
}

Juan C. Miñano, Rubén Mohedano, Pablo Benítez, and Roberto Alvarez

Low-cost, plastic-injected optics mix light from different color LED dies without a significant decrease in average brightness, simplifying luminaire design both optically and electronically.

In solid-state lighting, high-flux and high-color rendering index (CRI) light engines may be achieved by arraying and mixing the light from different color dies or phosphors, or a combination of the two, in the LED package. However, these nonhomogeneous sources, when combined with luminaire optics, tend to produce patterns with undesirable artifacts such as spatial and angular nonuniformities and color separation (see Figure 1).

To preclude these effects, the light should be mixed with both luminance and spectral considerations in mind. While diffusers can achieve this mixing, they also increase the apparent size of the source (étendue), reducing its average brightness and resulting in optical systems that are either larger (if the output beam angles have to be preserved) or have wider emission angles (if luminaire size is limiting). In addition, diffusers producing medium-angle scattering, needed to achieve smooth color mixing, tend to be inefficient because the light output ratio of a diffuser rarely exceeds $80 \%$ owing to backscattering and absorption.

To address these issues, we developed the Shell Mixer, a small optic with a diameter two to three times the overall LED size and comprising mini-lenses arranged on both the outer and the inner surfaces of a thin hollow dome covering the source (see Figure 2). ${ }^{1}$ These mini-lenses perform Köhler integration, ${ }^{2}$ an optical arrangement that homogenizes illumination in microphotography, by both spatially and angularly mixing the light emitted from the integration zone near the dome's center. We determined that placing the dome on top of a multi-die Lambertian light source produces a highly homogeneous virtual source that appears to lie on the plane of the dies. As this arrangement has essentially the same overall emitting area as the real source (the die cluster), it does not severely decrease the average brightness. Thus, we demonstrated that the addition of our Shell Mixer,

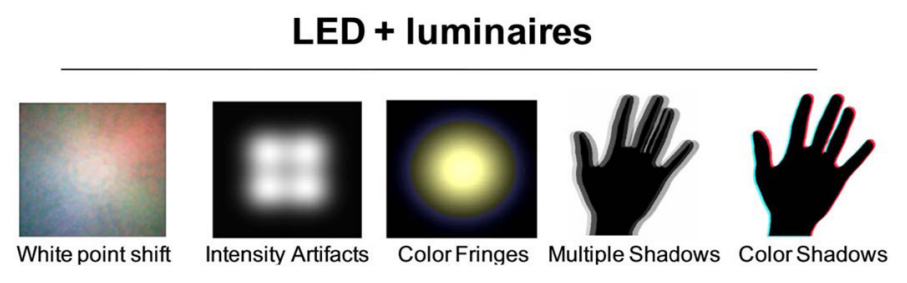

Figure 1. The undesirable results produced by using multi-die (and especially multi-color) packages with typical luminaires.

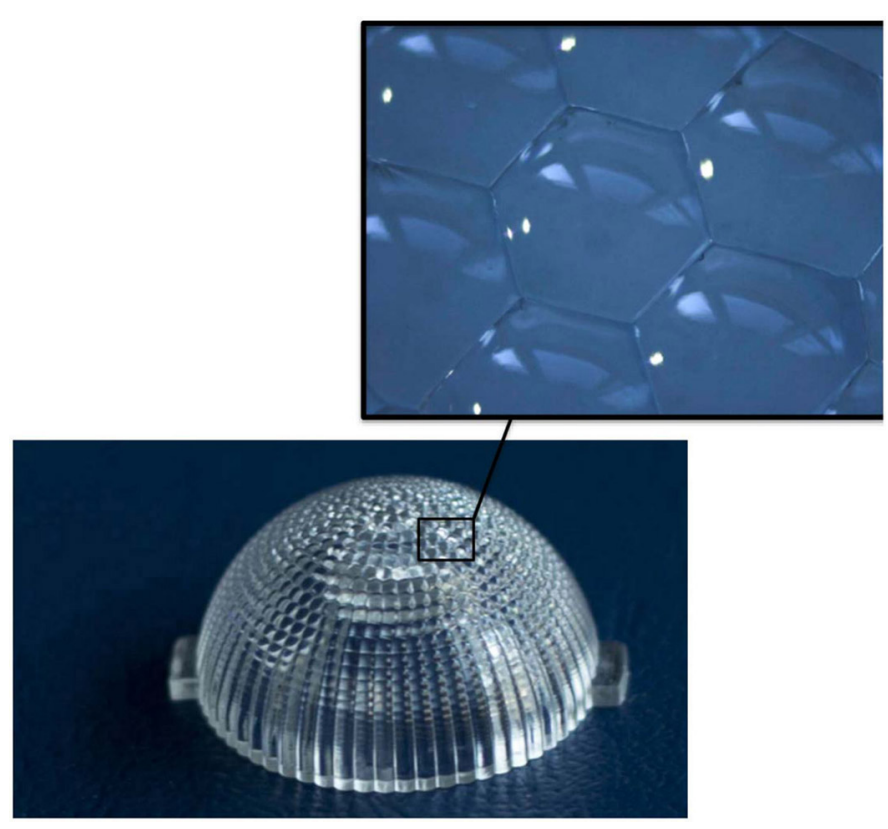

Figure 2. A 28mm-diameter Shell Mixer prototype with a detail of the micro-lenses that are on the outside surface of the shell.

together with the original LED and secondary optics, automatically changes the performance of the luminaire and results in a homogeneous pattern without color separation. 

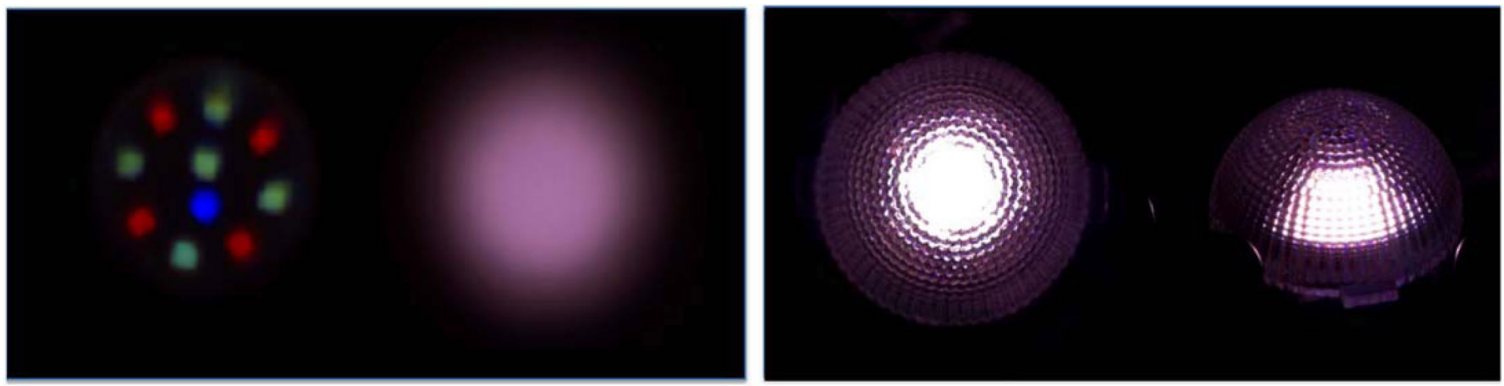

Figure 3. On the left is an image of the multi-color light source without and with the Shell Mixer, as projected by a lens. On the right is a multi-color light source as seen through the Shell Mixer from the top $\left(90^{\circ}\right)$ and from the side $\left(45^{\circ}\right)$.
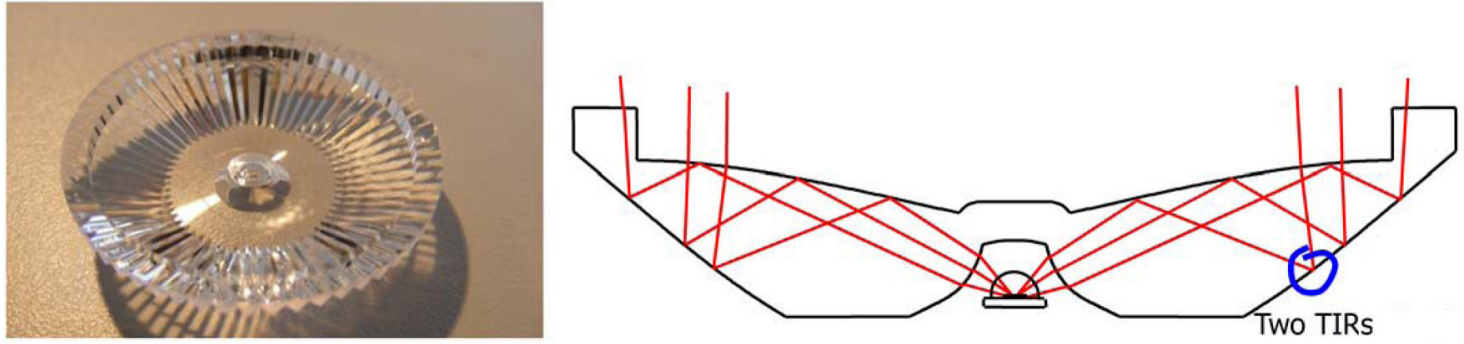

Figure 4. The V-groove RXI is an ultra-compact lens (its depth being one fourth of its diameter) with good collimation and mixing skills, making this device compatible with spotlight applications. The V-groove RXI does not need metal coatings because the light is bounced by two total internal reflections (TIRs) on the back surfaces.

Additionally, light bouncing off the hemispherical shell will land in the vicinity of the dies and will have a second chance to be reflected and extracted, especially when this vicinity is colored white. Compared to other devices based on lenses, our Shell Mixer offers a simple optical approach to creating a universal light engine. Moreover, experimental measurements of our first Shell Mixer prototypes, developed under the scope of a European Union 7th Framework Programme project, SSL4EU, ${ }^{3}$ have shown the device's optical efficiency to be above $94 \%$, in good agreement with ray-tracing simulations (see Figure 3).

Another way to produce good mixing is so-called anomalous deflection, ${ }^{4}$ by which the luminaire optics can produce the required beam pattern and light mixing at the same time. Our V-groove RXI collimator is among the devices that have such capability. This simultaneous-multiple-surfaces (SMS) ${ }^{5,6}$ ultracompact device has excellent collimating properties and is compatible with spotlight applications. Its shallow profile arises from the double use of its front optical surface, which first bounces the light coming from the LED toward a grooved back mirror and later refracts the light coming from this grooved mirror (see Figure 4).

We designed the back mirror of the V-groove RXI device to feature a set of free-form grooves that bend the light by means of two total internal reflections (TIRs) that mix the light. As there are no metal coatings, injection-molding technology can mass manufacture the optic very inexpensively. Figure 5 compares our V-groove RXI to a conventional metallized one. The figure also shows both the rotationally symmetric V-groove RXI and a freeform cropped version that further enhances the device's blending capabilities by adding aplanatic features to its symmetric counterpart with no loss in efficiency.

Good light mixing is important not only for simplifying luminaire optical design but also for obviating die binning, and it dramatically simplifies dimming or color tuning electronics. We have shown that when a good optical light mixer is used, simply switching off some of the dies in the array can achieve these two functions without affecting the emission pattern. Moreover, optical light mixing may reduce cost by avoiding pulse-width modulation and other patented electronic solutions for dimming and color tuning.

We are now investigating further improvements in light mixing. Our main goal is to increase the average radiance of the mixed light while keeping a high mixing quality (such as the one shown in the free-form cropped V-groove RXI of Figure 5),

Continued on next page 


\section{Mirror-based RXI}
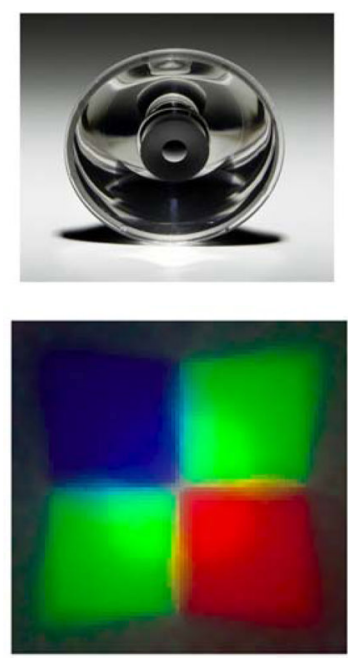

V-groove RXI
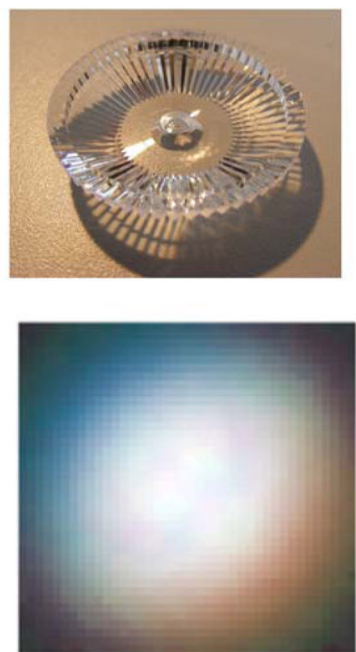

8-fold free form V-groove RXI
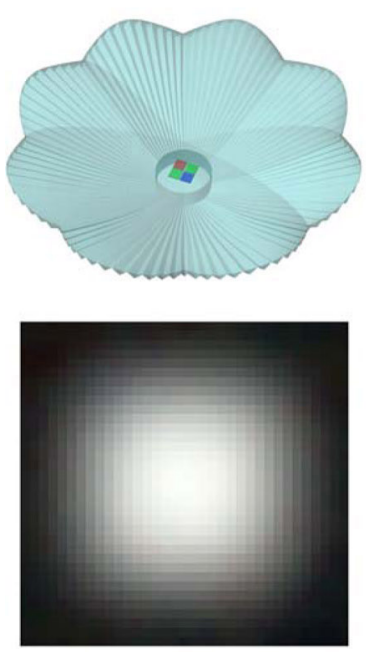

Figure 5. Three versions of the RXI optic. Left: The original RXI has mirror coatings and excellent collimation capabilities. Its far-field spot is an image of the light source. Center: The V-groove RXI version offers the same collimation features, but because it does not form an image of the light source, it produces a degree of mixing that negates some of the need for LED chip binning. Right: An advanced version of the latter is the multi-fold V-groove RXI, which can collimate and mix light at the same time.

and, to keep the cost of optics low, avoiding the use of dichroic (interferential) mirrors.

\section{Author Information}

\section{Juan C. Miñano and Pablo Benítez}

Polytechnic University of Madrid (UPM)

Madrid, Spain

Since 1982, Juan C. Miñano has been involved in nonimaging optics and has developed several optical design techniques with immediate application to optoelectronics, such as the SMS method. Since 1997, he has been a professor at UPM, and since 2000 he has collaborated with Light Prescriptions Innovators (LPI) as senior scientist.

Pablo Benítez received his PhD in 1998, having first received a BS in telecommunication engineering from UPM in 1993 and in mathematics from the Universidad Complutense de Madrid that same year. He has been an associate professor at UPM since 1999 and an associate scientist at LPI. His research ranges from optical design to manufacturing and applications, and he is a co-inventor of the SMS optical design method.

\author{
Rubén Mohedano and Roberto Alvarez \\ LPI \\ Altadena, CA \\ and \\ Madrid, Spain
}

Rubén Mohedano obtained his PhD in January 2002, focusing on nonimaging optics design and manufacturing. He is currently managing director of the LPI office in Europe, a research and development company he joined in 2002. He has participated in several major illumination, automotive, and concentration photovoltaic projects for various companies.

Roberto Alvarez has an MBA in finance from the Wharton School, University of Pennsylvania, and a BS in engineering from the University of California, Los Angeles. He has over 30 years of entrepreneurial and management experience and has led several firms involved in solar energy, solar micro-utilities, and energy conservation. In addition to his varied experience in developing thermal solar collectors, mainframe computers, computer peripherals, and other electromechanical devices, he has been a co-inventor of several patented products in the nonimaging optics field. 


\section{References}

1. J. Chaves, A. Cvetkovic, R. Mohedano, O. Dross, M. Hernández, P. Benítez, J. C. Miñano, and J. Vilaplana, Inhomogeneous source uniformization using a shell mixer Köhler integrator, Proc. SPIE 8550, p. 85502X, 2012.

2. O. Dross, R. Mohedano, M. Hernández, A. Cvetkovic, P. Benítez, and J. C.

Miñano, Köhler integration optics improve illumination homogeneity, Laser Focus World, May 2009. doi:10.1117/12.798111

3. http://ssl4.eu Solid State Lighting for Europe. Accessed 16 February 2013.

4. P. Benítez, J. C. Miñano, A. Santamaría, and M. Hernández, On the analysis of rotational symmetric microstructured surfaces, Opt. Express 15 (5), pp. 2219-2233, 2007. doi:10.1364/OE.15.002219

5. J. C. Miñano, J. C. González, and P. Benítez, A high-gain, compact, nonimaging concentrator: RXI, Appl. Opt. 34 (34), pp. 7850-7856, 1995. doi:10.1364/AO.34.007850 6. R. Winston, J. C. Miñano, and P. Benítez, Nonimaging Optics, Academic Press, Boston, 2005 